\title{
estructuras metálicas ligeras
}

\author{
Conferencias pronunciadas en el aula de Costillares por el profesor \\ Dr. HERMANN BEER, GRAZ - AUSTRIA
}

$846-1$

\author{
La 1.a parte de este trabajo se publié \\ en el múmero 193 de esta Revista
}

Si se considera una barra (por ejemplo de un sistema reticulado) formada por un perfil I (dos perfiles II con almas unidas mediante soldadura por puntos) o un perfil angular (figura 34) pueden dibujarse los diagramas del pandeo de la chapa (pandeo local) como función de la relación del ancho de las alas al espesor de la chapa $\left(b_{0} / t\right)$. Se han calculado para un acero de construcción con límite de fluencia de $2.300 \mathrm{~kg} / \mathrm{cm}^{2}$ y la tensión básica, admisible, de 1.400 kilogramos $/ \mathrm{cm}^{2}$, las tensiones críticas $(A, B$, $C$ ) y las tensiones admisibles considerando el pandeo local $(a, b, c)$. Puesto que el perfil I (1) dispone de una reserva postcrítica relativamente grande, hemos calculado también la curva $(D)$, que representa el límite superior de la resistencia

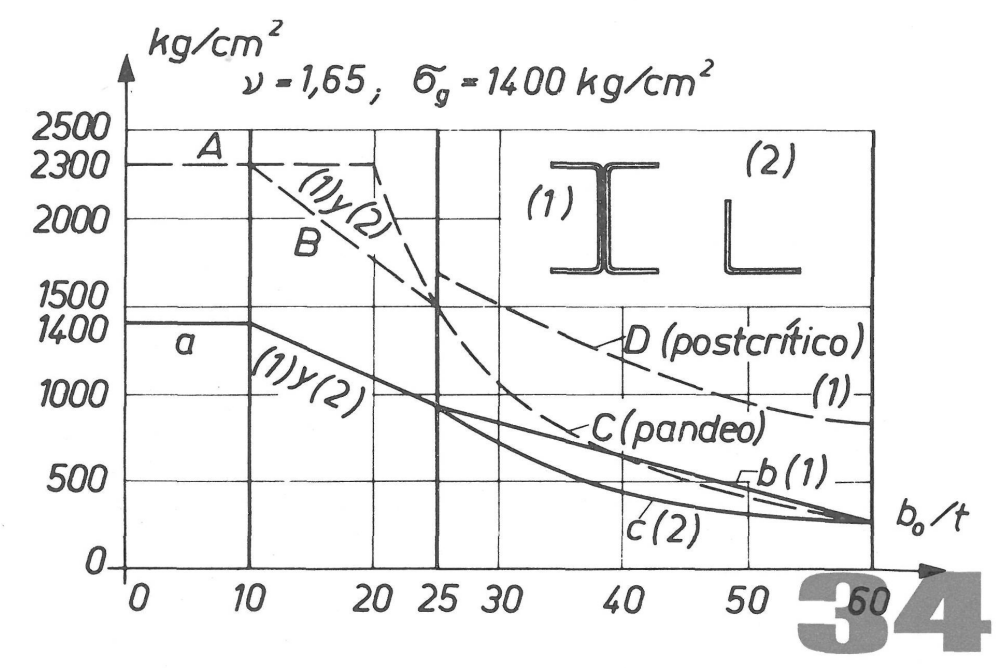

de la chapa. Podemos admitir en este caso una tensión elevada bajo carga de servicio (curva $b 1$ ). Sin embargo, para el perfil $L$ esta reserva postcrítica es relativamente pequeña, particularmente en la zona $b_{0} / t$ cercana a 25 , porque este perfil pierde su resistencia por 
el pandeo con torsión de la barra. Se ha calculado por esto la curva $C 2$ de las tensiones admisibles del perfil $L$, empezando con una seguridad $v=1,65$ contra el pandeo y disminuyéndola hasta llegar a $v=1$ para $b_{0} / t=60$. Partiendo de chapas gruesas, se observa que el límite de fluencia vale solamente hasta la relación $b_{0} / t=10$, seguida del descenso de la curva de pandeo. Teniendo un diagrama de tensiones con fluencia acusada (véase figura $1 a$ ) puede tomarse el límite de fluencia hasta $b_{0} / t=20$, pero ya diferencias pequeñas en el recorrido de este diagrama tienen influencia en la resistencia al pandeo, y por esto se ha conservado como límite el valor $b_{0} / t=10$.

Los diagramas discutidos demuestran claramente que es muy antieconómico emplear perfiles con alas muy delgadas, porque la tensión admisible disminuye rápidamente para relaciones $b_{0} / t>20$. Para relaciones $b_{0} / t>15$ se recomienda reforzar las alas con «labios».

Ya hemos dicho que es imprescindible considerar en una barra comprimida, fuera del pandeo local de la chapa, también el pandeo de la barra con su longitud total. Es, por esto, muy interesante calcular la curva que da la relación $b_{0} / t$ como función de la esbeltez, teniéndose en este caso la misma seguridad contra pandeo de la barra y pandeo local. En la figura 35 se dan estos valores $b_{0} / t$ para dos relaciones del ancho de las alas a la altura del alma, a saber: $b=0,4 h$ y $b=h$ en los aceros St 37 y St 52 . Se nota que en las zonas elásticas $(\lambda>108$ para St 37 y $\lambda>85$ para St 52) la relación entre $\lambda$ y $b / t$ es lineal, mientras que para esbelteces más pequeñas las rectas se transforman en curvas cóncavas, lo que significa un leve aumento de la relación $b / t$. Resalta la diferencia entre St 37 y St 52, siendo antieconómico emplear aceros de alta resistencia para la zona elástica. Un perfil (St 37) con, por ejemplo, $b=0,4 h$, puede aprovecharse completamente al pandeo por flexión para $\lambda>50$, no necesitándose tener en cuenta el pandeo de las alas. Para St 52 este límite está cerca de $\lambda=62$. Teniendo un perfil con $b=h$, estos valores llegan a 75 y 90 , respectivamente. Es muy claro que la solución óptima se encuentra en la misma seguridad contra pandeo local y pandeo de la barra, lo que corresponde a las curvas presentadas.

Otra representación (fig. 36), muestra que es posible encontrar una sola curva comprendiendo todas las relaciones $b / h$. Se calcula para esto un valor auxiliar $k$ que permite determinar, con las fórmulas indicadas en la figura, la relación $b / t$ en función de la esbeltez.

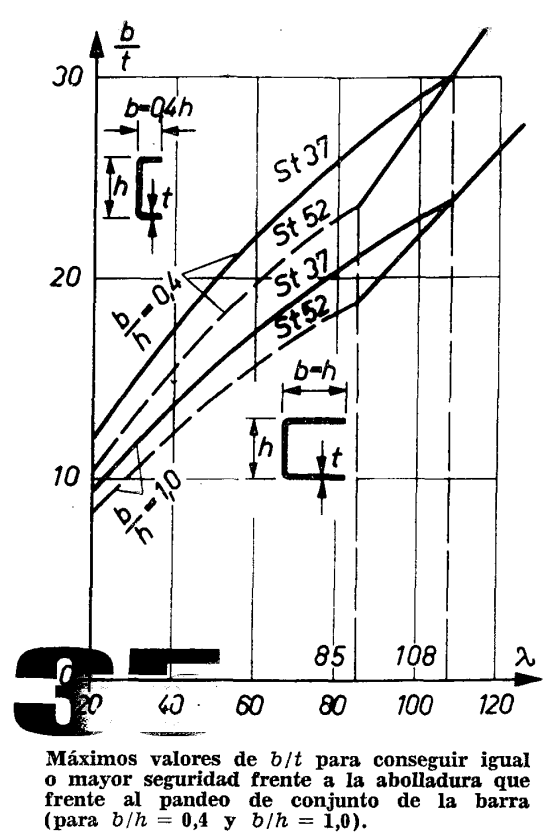
Fíjense ustedes en el salto de la curva para $b / h=0,326$, lo que corresponde al límite entre pandeo del ala (ancho $b$ ) y pandeo del alma (altura $h$ ), siendo en el primer caso $h / t=C \sqrt{k}$, y en el segundo caso $b / t=$ $=C \sqrt{k}$. Con este diagrama puede calcularse rápidamente la relación $b / t$ y la esbeltez $\lambda$, obteniendo así seguridades iguales frente al pandeo local y al pandeo de la barra.

En sistemas de celosías se usa frecuentemente el perfil angular de paredes delgadas para diagonales y montantes, como así también para cordones, por ejemplo en torres de líneas eléctricas (fig. 37). Suponiendo la barra bajo compresión axil, se necesita investigar, fuera del pandeo por flexión perpendicularmente al eje de $i_{\min }$, el pandeo por torsión. Comparando la fórmula para este último caso con la fórmula para el pandeo local de una sola ala, se ve que ambas expresiones difieren solamente en un factor $\left(1-v^{2}\right)$ en el primer término del paréntesis, siendo $\nu=0,3$ la constante de Poisson (fig. 37). Poniendo $0,91 \approx 1$ se obtiene la coincidencia de ambos casos. Los ensayos han demostrado que la resistencia de la 

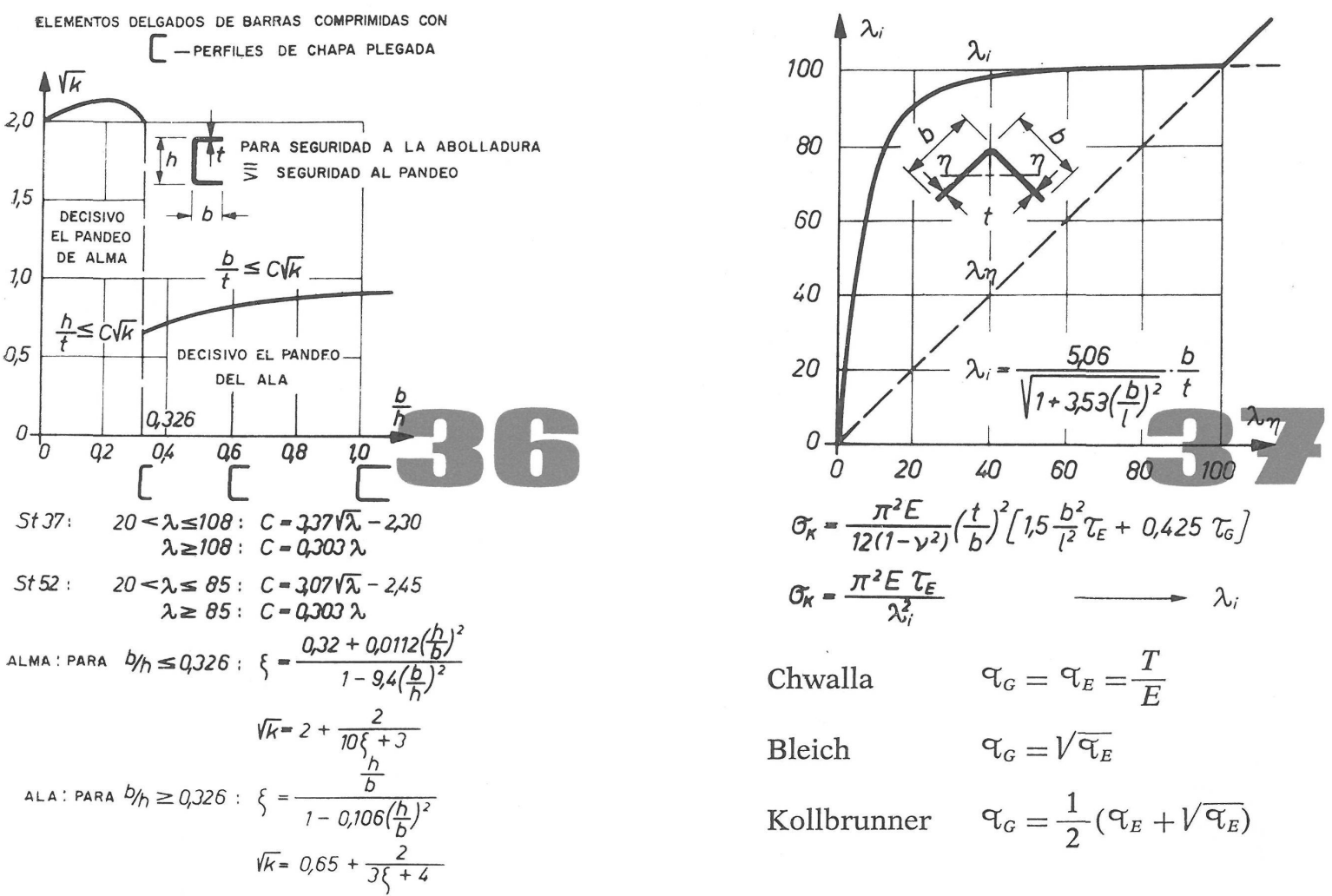

$$
\begin{aligned}
& \sigma_{K}=\frac{\pi^{2} E}{12\left(9-\nu^{2}\right)}\left(\frac{t}{b}\right)^{2}\left[1,5 \frac{b^{2}}{l^{2}} \tau_{E}+0,425 \tau_{G}\right] \\
& \sigma_{K}=\frac{\pi^{2} E \tau_{E}}{\lambda_{i}^{2}} \longrightarrow \lambda_{i}
\end{aligned}
$$$$
\text { Chwalla } \quad q_{G}=q_{E}=\frac{T}{E}
$$$$
\text { Bleich } \quad q_{G}=\sqrt{\tau_{E}}
$$$$
\text { Kollbrunner } \quad q_{G}=\frac{1}{2}\left(q_{E}+\sqrt{q_{E}}\right)
$$

barra se agota por el pandeo de las alas en toda la longitud de la barra. Sin embargo, la arista de las alas no queda recta, sino que toma una leve curvatura. El segundo término de la fórmula indicada da la influencia de la torsión. La relación $q_{G}$ de los módulos tangenciales en las zonas plástica y elástica, respectivamente, depende de la tensión de compresión. Mientras que E. Chwalla supone para $q_{G}$ el valor $q_{E}=\frac{T}{E}$, siendo $T$ el módulo de pandeo de Engesser-Shanley, F. Bleich propone $q_{G}=\sqrt{q_{E}}$, y, finalmente, Kollbrunner da una solución intermedia con $q_{G}=\frac{1}{2}\left(q_{E}+\sqrt{q_{E}}\right)$.

El valor de Chwalla queda del lado más seguro. La curva da la esbeltez ficticia con la cual debe calcularse el pandeo de la barra perpendicularmente al eje $i_{\min }$, no necesitándose tener en cuenta el pandeo por torsión. La línea de trazos significa la esbeltez para el pandeo por flexión solamente. Considerando el diagrama, vemos claramente que el pandeo por torsión reduce mucho la resistencia a la compresión de un perfil angular. Teniendo, por ejemplo, una esbeltez $\lambda_{\eta}=50$, se necesita calcular el pandeo con el doble de esta esbeltez $\left(\lambda_{i}=100\right)$. Con $\lambda>100$ se pasa a la zona elástica, necesitándose considerar solamente el pandeo por flexión con $\lambda_{\eta}$.

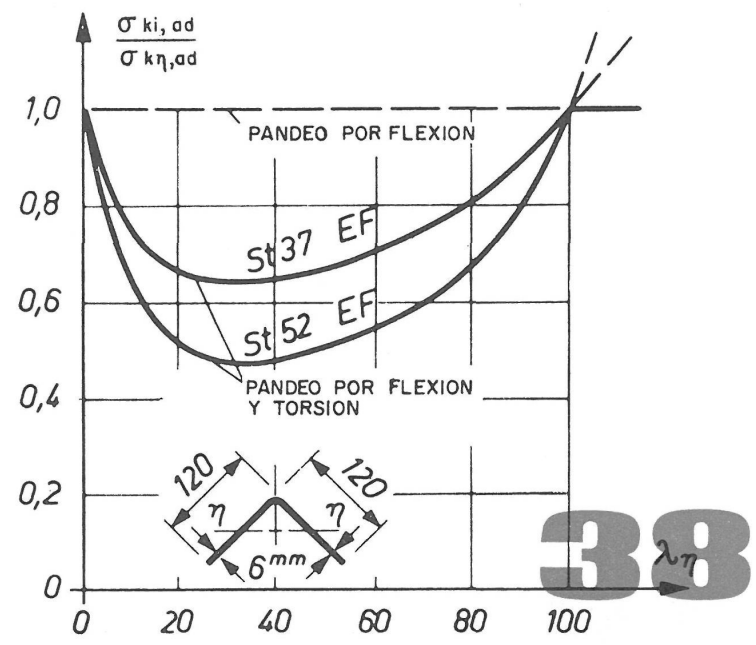




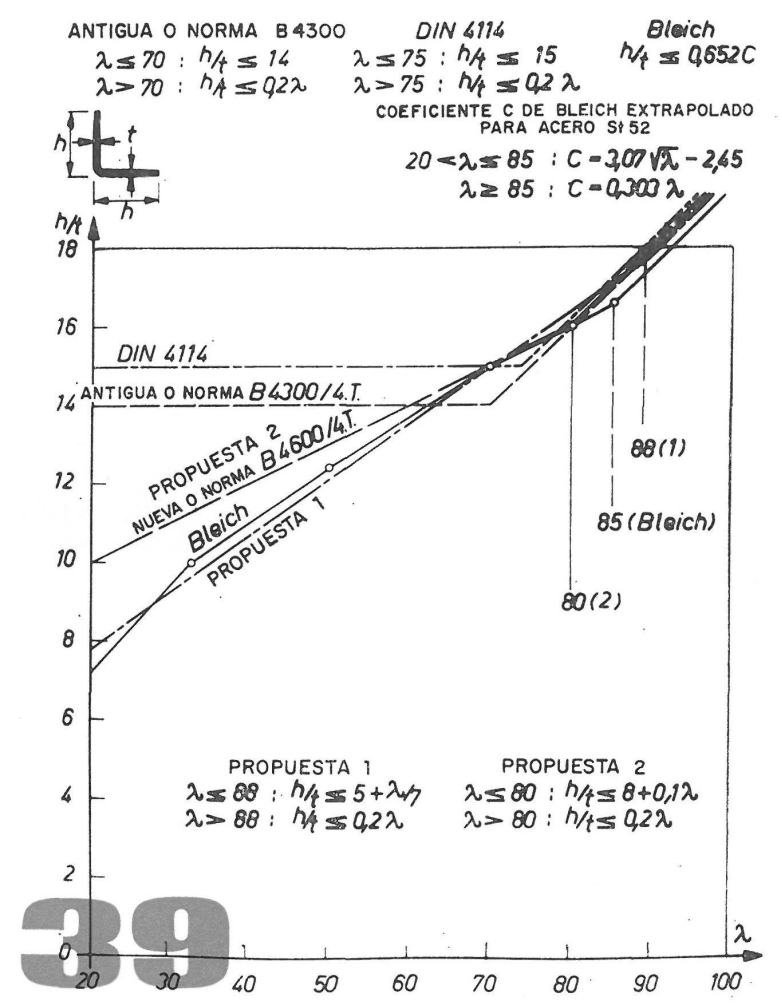

Elementos delgados de las piezas comprimidas.
En torres de líneas de alta tensión se usa frecuentemente el perfil angular $120 \times 120 \times 6$, fabricado por laminación o plegado en frío. La figura 38 muestra la relación de la tensión ficticia admisible para el cálculo de pandeo $\sigma_{k i, a d}$ (considerando también el pandeo por torsión) a la tensión de pandeo por flexión $\sigma_{k, a d}$. La línea de trazos da los valores para pandeo por flexión simple. Suponiendo una barra de acero 52 con una esbeltez de $\lambda=60$, se reduce la tensión admisible para el dimensionado a la mitad del valor correspondiente al pandeo simple por flexión. Es por esto muy antieconómico emplear el perfil $L 120 \times 120 \times 6$ para esbelteces que se encuentran en la zona plástica $\left(\lambda_{\eta}<100\right)$, sin prever medidas para aumentar la seguridad al pandeo de las alas. Volveré más adelante sobre este problema.

Las Normas austríacas prestaron mucha atención al problema de pandeo por torsión de perfiles angulares. En la figura 39 están representadas las Normas antiguas austríacas y la DIN 4114, que suponen constantes las relaciones $h / t$ para esbelteces menores de 70 y 75 , respectivamente. Para esbelteces que sobrepasan este límite, se toma una relación lineal con $h / t=0,2 \lambda$. Según investigaciones de Bleich se obtiene el polígono de la figura sustituido aproximadamente por la recta $h / t=5+\frac{\lambda}{7}$ (propuesta 1). La nueva ÖNORM levantó esta recta un poco, obteniendo la recta menos inclinada $h / t=80,1 \lambda$ hasta $\lambda=80$, continuada por la recta $h / t=0,2 \lambda$ (propuesta 2 ).

Trataremos ahora el problema de reforzar el perfil angular contra el pandeo por torsión, sin aumentar el espesor de las alas, eligiendo como ejemplo el perfil $L 120 \times 120 \times 6$. Puede
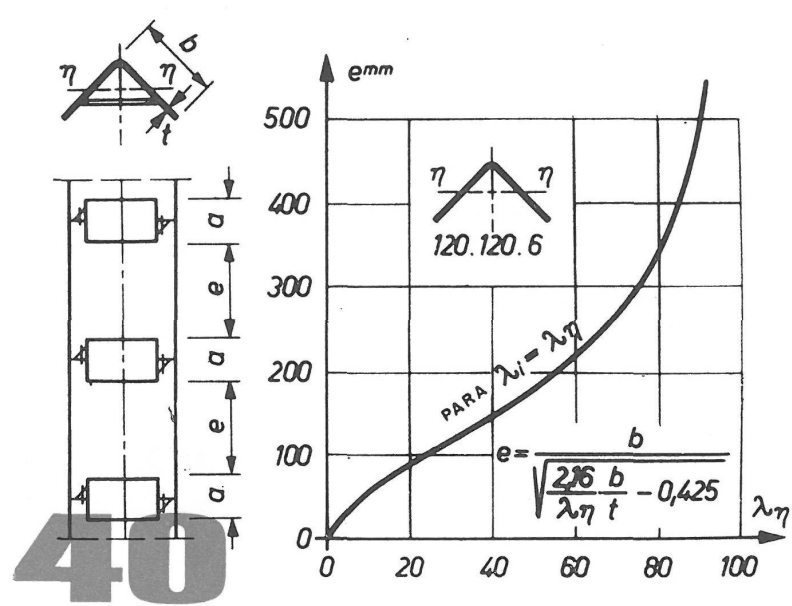
pensarse en soldar presillas entre las alas (fig. 40), pero solamente con separaciones muy cortas se logra un aumento de la seguridad al pandeo en la zona más importante de las esbelteces. El diagrama da la separación $e$ de presillas en función de la esbeltez $\lambda_{\eta}$ considerando el pandeo simple. Se ve que, teniendo una esbeltez de $\lambda_{\eta}=60$, se obtiene una $e$ igual tan sólo a dos veces el ancho de las alas, siendo por esto antieconómica esta medida.

Una medida más eficaz es la disposición de «labios» en las alas, como puede verse en la figura 41 . Pueden confor- 
marse fácilmente estos labios mediante el proceso de plegado en frío. Se han comparado mediante los diagramas de la figura los casos 1 (sin labios), 2 (con labios hacia afuera) y $\mathbf{3}$ (con labios hacia adentro), suponiendo la misma área de sección. Se ve que se reduce mucho la esbeltez ficticia para el dimensionado colocando labios. Teniendo, por ejemplo, una relación de longitud de la barra al ancho de las alas $l / b=10$, se obtiene con el perfil liso una esbeltez ficticia de $\lambda_{i}=107$, reduciéndose esta esbeltez en el perfil con labios hacia adentro a $\lambda_{i}=75$. Tratándose de cordones para torres reticuladas debe pensarse en la unión de las diagonales con las alas, colocándolas por fuera o por dentro según la posición de los labios.

Se obtiene una solución muy económica para igualar en lo posible las seguridades contra pandeo por flexión y torsión, respectivamente, soldando una barra redonda de sólo 6 milímetros en zigzag, en los lados internos de las alas (fig. 42). En el caso considerado en la figura se necesita tener en cuenta el pandeo por torsión solamente para $\lambda_{\eta}<58$ (siendo: $\lambda_{\min }=2,92 b / t$ ). En este caso se supone para el cálculo que el ala está empotrada en la arista.

Podemos aumentar finalmente la seguridad al pandeo por torsión colocando un tubo, de tal manera que se tenga bastante lugar para la unión de la diagonal; sin embargo, esta medida no tiene mucha importancia práctica.

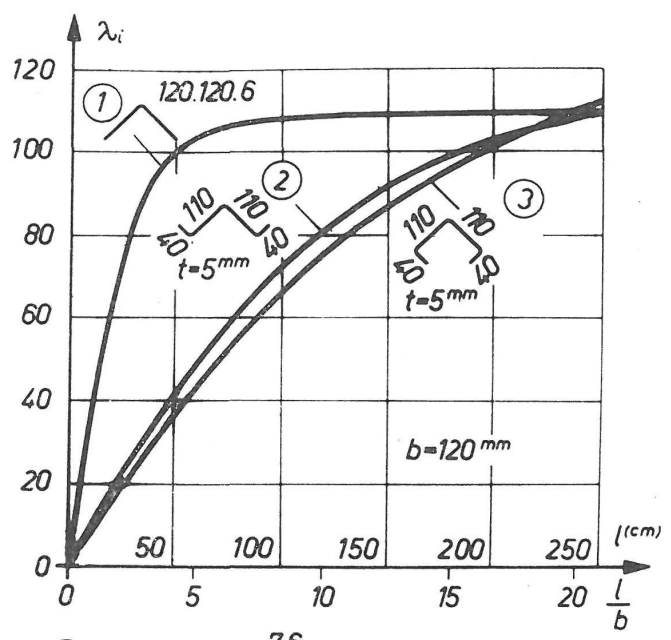

(1) $\lambda_{i}=\frac{7,6}{\sqrt{1,51 / 1^{2}+0,00493}}$

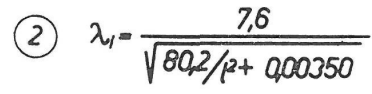

(3) $\lambda_{i}=\frac{7,6}{\sqrt{102 / 12+0,00307}}$

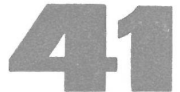

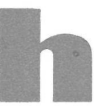

La viga de alma ulema

Si se considera el pandeo del alma en una viga, se tiene casi siempre una combinación de pandeo por flexión y por esfuerzo cortante (fig. 43). Las Reglas americanas «Specifica-

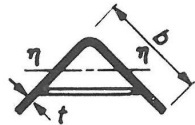

ABOLLADURA

$\alpha=\frac{b}{e} \geq 263 \sqrt[4]{\tau_{E}}$

$k_{\text {min }}=1,28$

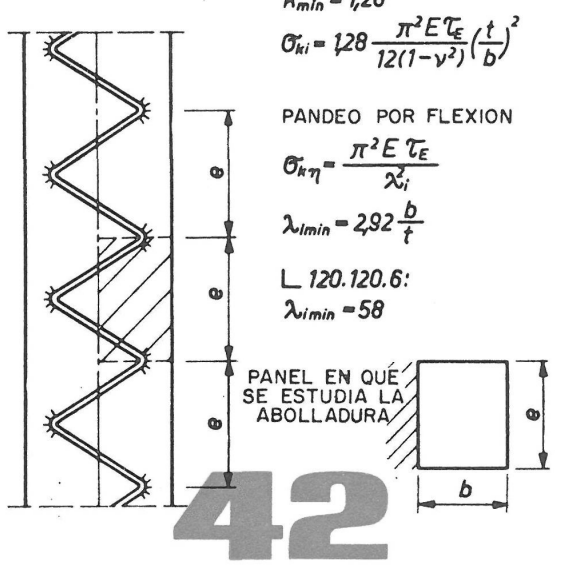

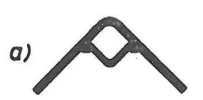

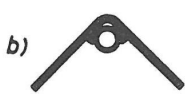

c)
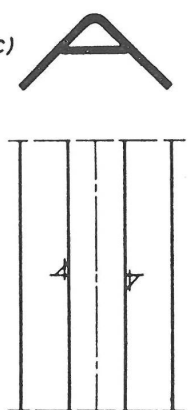
tion for the Design of Light Gage Cold Formed Steel Structural Members» presentan fórmulas aproximadas para los dos casos de pandeo, suponiendo el alma sin rigidizadores transversales. La tensión crítica para el esfuerzo cortante se fija en dichas Reglas:

$$
\tau_{c r}=\left(t / h_{s t}\right)^{2} \times 10^{7}
$$

y la tensión admisible:

$$
q_{a d}=\left(t / h_{s t}\right)^{2} \times 4,5 \times 10^{6} .
$$

Para flexión pura se tiene:

$$
\sigma_{c r}=\left(h_{s t} / t\right)^{2} \times 45 \times 10^{6}
$$



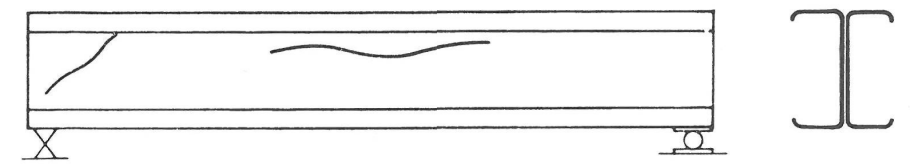

alma: $\tau_{c r}=\frac{90^{7}}{\left(h_{s+1}(t)^{2}\right.}$

$$
\tau_{\text {ad }}=\frac{4,5 \cdot 10^{6}}{\left(h_{s t} / t\right)^{2}} \equiv \frac{2}{3} \sigma_{g}
$$

$$
\sigma_{c r}=\frac{45 \cdot 10^{6}}{\left(h_{s t} / t\right)^{2}}
$$$$
\sigma_{c d}=\frac{36,6 \cdot 0^{6}}{\left(h_{s t} / t\right)^{2}}<\sigma_{9}
$$

\section{fórmula de interacción.}

$$
\left[\frac{\sigma_{\text {Dexist }}}{\sigma_{\text {Dad }}}\right]^{2}+\left[\frac{\tau_{\text {exist }}}{\tau_{\text {ad }}}\right]^{2}=1
$$

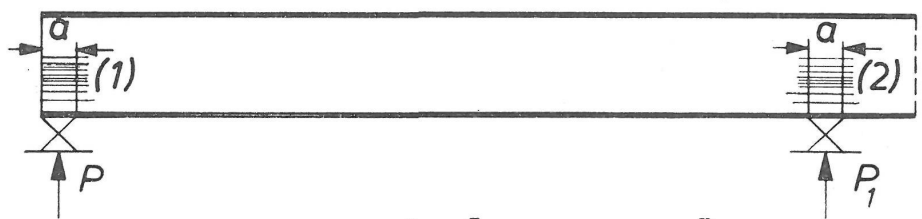

$$
\left\{\begin{array}{l}
\text { (1) } P_{\text {máx }}=t^{2} \sigma_{g}\left[7,4+0,93 \sqrt{\frac{a}{t}}\right] \\
\text { (2) } P_{\text {máx }}=t^{2} \sigma_{g}\left[11,1+2,41 \sqrt{\frac{a}{t}}\right]
\end{array}\right.
$$
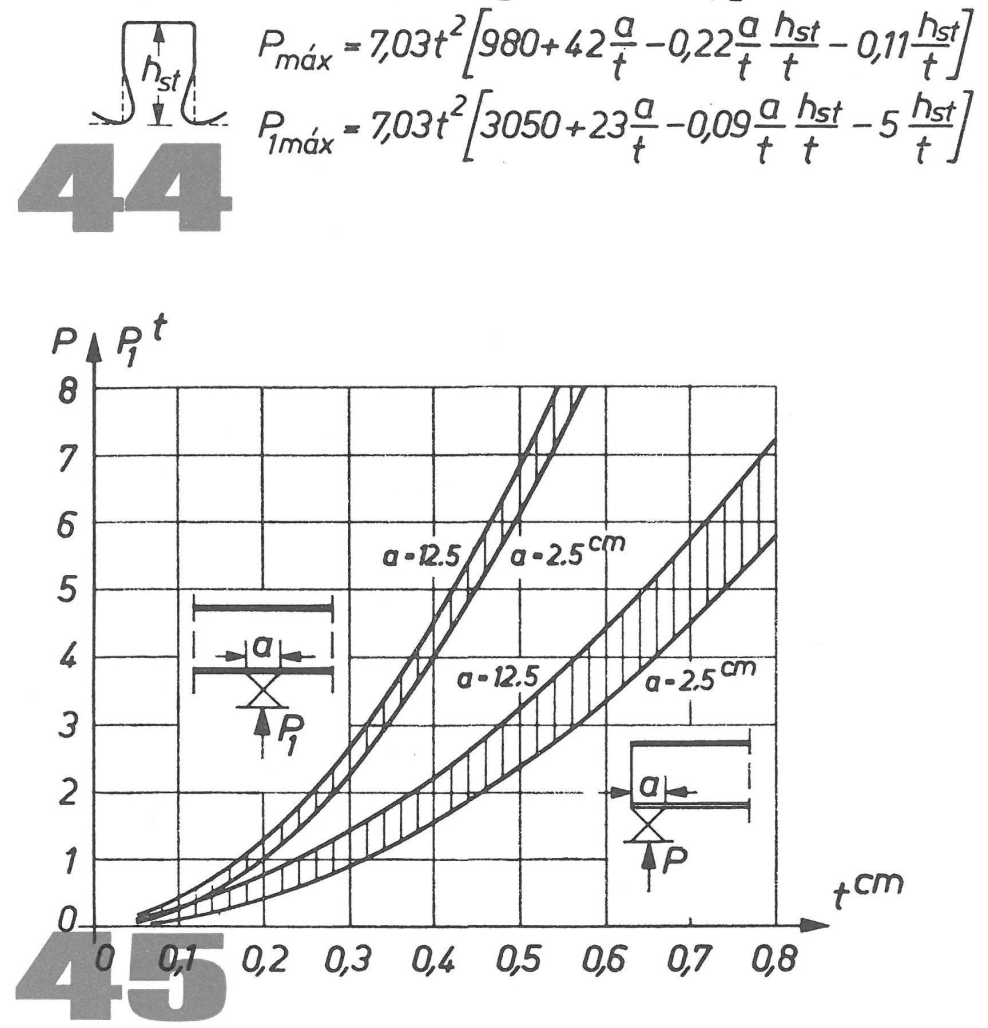

54 y la tensión admisible correspondiente:

$$
\sigma_{a d}=\left(h_{s t} / t\right)^{2} \times 36,6 \times 10^{6} .
$$

Además, se exige una seguridad mínima de 1,65 frente a $2 / 3 \sigma_{F}$ para solicitaciones de esfuerzo cortante y frente a $\sigma_{F}$ para solicitaciones de flexión, respectivamente. Ustedes pueden observar que los coeficientes de seguridad al pandeo difieren según el carácter de la solicitación. Para el esfuerzo cortante puro se toma 2,2 y para flexión pura 1,23, justificándose esta diferencia con la mayor reserva postcrítica que se tiene bajo flexión, a causa de la posibilidad de trasposición de las tensiones a las zonas próximas al cordón superior. Para la solicitación al corte, el alma pandeada no dispone ya de reservas, y una abolladura inicial, aunque sea leve, puede favorecer el pandeo. Sin embargo, para relaciones $h_{s t} / t<90$, puede reducirse el factor de seguridad hasta llegar a 1,65 para $h_{s t} / t=45$.

El límite superior para la validez de las fórmulas indicadas es de $h_{s t} / t=150$.

Para la acción conjunta de ambos tipos de solicitaciones vale la fórmula de interacción:

$$
\left[\frac{\sigma_{e x}}{\sigma_{a d}}\right]^{2}+\left[\frac{\tau_{e x}}{\tau_{a d}}\right]^{2}=1
$$

necesitándose comprobar además que:

$$
\tau_{a d} \bar{\gtrless} \frac{2}{3} \times \frac{\sigma_{F}}{1,65} \quad \text { y } \quad \sigma_{a d} \bar{\gtrless} \frac{\sigma_{F}}{1,65} .
$$

Las cargas concentradas pueden causar un plegado del alma en la zona del punto de aplicación de la carga. La investigación teórica de este fenómeno de cedimiento por dobladura (en inglés: "crippling», en alemán: «krüppeln») es muy compleja. Ensayos norteameri- 
canos posibilitaron el desarrollo de una fórmula empírica para calcular la fuerza admisible $P_{\max }$, a fin de evitar el plegado del alma. Hay que distinguir dos casos distintos de vigas (fig. 44): El primero corresponde a la sección I, compuesta de dos perfiles $]$ [ con almas unidas. El plegado del alma está impedido parcialmente por el empotramiento en la base. En el segundo caso se tiene una sección consistente de dos almas, formada, por ejemplo, por un perfil del tipo "sombrero». Aquí no puede producirse un efecto de empotramiento del alma en su base. La reacción de apoyo (u otra carga concentrada) $P_{\max }$ depende, además de la tensión admisible básica, también del espesor de la chapa y de la longitud del apoyo. Pero existe también una diferencia esencial entre apoyos en los extremos y apoyos intermedios, siendo los primeros más desfavorables. Por esto se presentan en la figura las fórmulas empíricas para ambos casos.

Los diagramas de la figura 45 muestran claramente esta diferencia. Se han dibujado las curvas de $P$ (apoyo en el extremo) y de $P_{1}$ (apoyo intermedio) en función del espesor del alma, eligiendo dos longitudes distintas del apoyo $(2,5$ y $12,5 \mathrm{~cm})$. Vean ustedes que prácticamente puede duplicarse la reacción $P$ a fin de obtener $P_{1}$, pero llama la atención que el valor de esta reacción no depende tanto como se podía esperar de la longitud de apoyo $a$. Llegamos a la conclusión de que la única solución eficaz para aumentar, en un caso dado, la reacción admisible, es reforzar esta zona colocando un alma más gruesa o un perfil con misión de rigidizador.

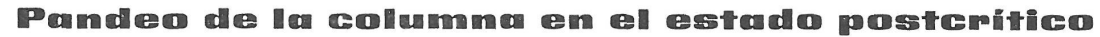

Consideramos ahora una columna con sección delgada, solicitada a la compresión. Si se excluye el pandeo por torsión, tenemos que tomar en cuenta solamente el pandeo local de las chapas y el pandeo de la barra alrededor de los ejes que correspondan a las esbelteces consideradas. Eligiendo el espesor de la chapa de tal manera que se tenga una seguridad al pandeo local $\nu_{B}=1$, obtenemos la curva superior de la familia de curvas dibujada en la figura 46. Admitiendo un pandeo local con una «seguridad» de solamente $\nu_{B}=0,8$, lo que equivale a decir que este pandeo tiene lugar cuando la carga real llegue al 0,8 del valor admisible, baja la curva de las tensiones admisibles, uniéndose con la primera curva en el punto $\lambda=135$. Si se reduce más aún el valor $\nu_{B}$, utilizando chapas más delgadas, se llega a valores de las tensiones admisibles muy bajos en la zona de esbelteces menores de 200 .

La familia de curvas de la figura 46 muestra claramente que debe elegirse cuidadosamente el espesor de pared para las columnas y las barras de las vigas de celosía, teniendo en cuenta que el óptimo se encuentra siempre en las zonas cercanas al empalme de las curvas con la correspondiente a $\nu_{B}=1$. Si se tiene plena libertad en la elección de la sección vale el principio de que, cuanto más se aumenta la esbeltez, más puede reducirse el espesor de la pared del perfil. Pero este ejemplo demuestra también claramente que debe considerarse siempre el problema del pandeo local conjuntamente con el pandeo de conjunto de la barra.

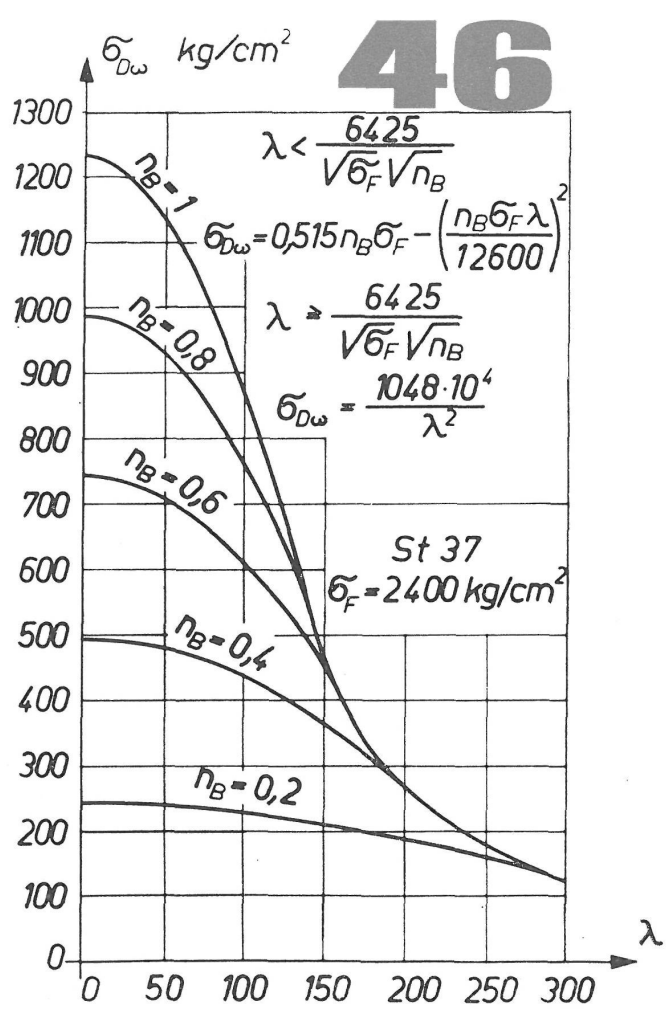




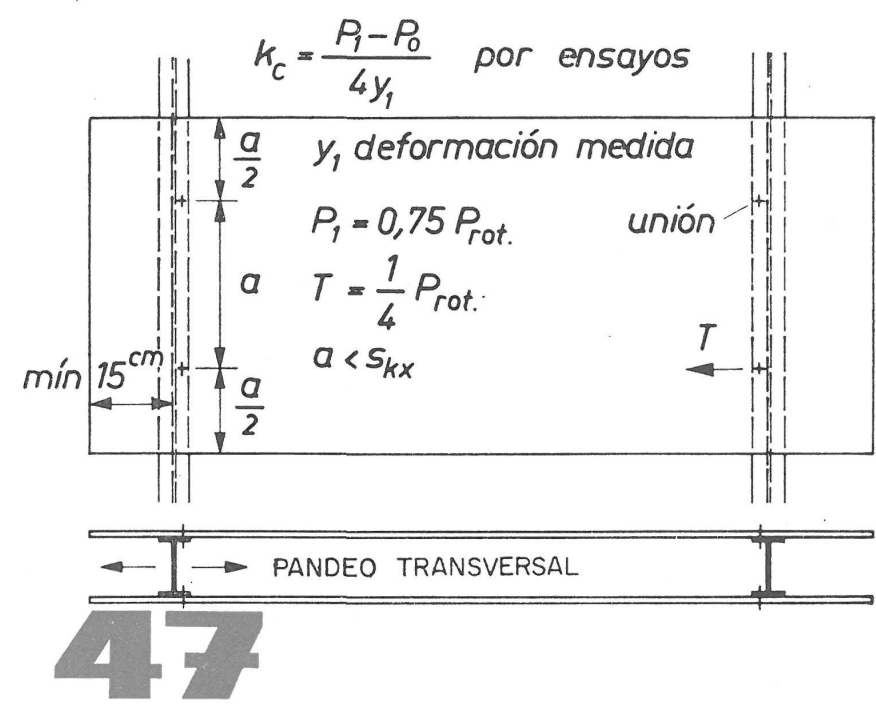

Las columnas intermedias de las estructuras de edificios tienen casi siempre un apoyo lateral en las paredes, aun cuando éstas sean paredes ligeras. A fin de evitar el pandeo de estas columnas en sentido transversal es necesario unir los paños de pared, cada cierta distancia, a las alas del perfil (figura 47). Para el cálculo de esta distancia es necesario conocer la constante de resorte $k_{c}$ del paño de pared unido con las alas; $k_{c}$ ( $\mathrm{kg} / \mathrm{cm}$ ) es la fuerza $T$, en $\mathrm{kg}$, que se necesita aplicar en la unión para causar un desplazamiento entre ala y pared de $1 \mathrm{~cm}$. Se ejecuta el ensayo con una sección de la pared unida con dos columnas, como se puede ver en la figura 47, suponiendo una fuerza inicial pequeña $P$ y aumentando esta fuerza hasta la rotura. Durante el proceso de carga se observa el desplazamiento $y$ entre pared y perfil, siendo finalmente $y_{1}$ el valor bajo la fuerza $P_{1}$ que corresponde al 75 por 100 de la fuerza de rotura. Con esta constante $k_{c}$ puede ejecutarse un cálculo del pandeo transversal de la columna según cualquiera de los procedimientos conocidos. Tomando para el dimensionamiento de la unión $T=\frac{1}{4} P_{\text {rot, }}$ se obtiene una seguridad más que suficiente para evitar el pandeo transversal.

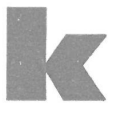

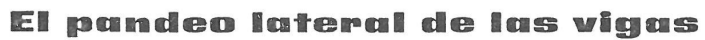

Considerando el pandeo lateral de las vigas, tenemos que observar que el peligro de pandeo lateral depende mucho del tipo de la sección. Mientras que para perfiles tipo "sombrero» no se presenta el pandeo lateral cuando se elige $J_{x}<J_{y}$ (véase la figura 48), cuando se trata de un perfil I o [ se presenta una reducción considerable de la tensión admisible a la flexión $\sigma_{a d, f l}$. En la figura se ha dibujado la curva de $\sigma_{a d, f l}$ en función de la esbeltez de la viga, considerando su eje $i_{\min }$. Se observa la gran reducción de la tensión admisible a la flexión, a causa del peligro de pandeo lateral, en la zona de esbelteces $\lambda>70$; pero llama la atención que el perfil I y el perfil [ tienen aproximadamente los mismos valores para $\sigma_{a d}, f l$. Sin embargo, el perfil 7 exige una reducción mucho mayor de $\sigma_{a d}$, $t$ como consecuencia de la forma de su sección, müy desfavorable frente al pandeo lateral.

Considerando estos diagramas, llegamos a la conclusión de que es necesario apoyar lateralmente el perfil I para aumentar su solicitación admisible a la flexión, ya que, en caso contrario, se obtiene una solicitación muy baja. Puede lograrse este apoyo mediante los elementos de cubierta o del forjado de piso, o por los elementos de cerramiento, aun cuando. estos elementos se compongan solamente de chapas de amianto-cemento o de madera. Para. luces mayores este apoyo es también necesario con vigas de secciones [ o I. Si no es posible colocar apoyos laterales, deben elegirse secciones en cajón o del tipo "sombrero».

Teniendo una viga de sección tipo «sombrero» que trabaje a la compresión en las alas pe-queñas, se necesita considerar el pandeo lateral de estas alas (fig. 49). Para evitar este peligro de pandeo puede considerarse el ala y una parte del alma como "barra» comprimida. apoyada elásticamente. Puede calcularse de manera aproximada la altura del alma que ac- 
túa conjuntamente con el ala con la fórmula: $\left(2 y_{d}-y_{z}\right)$.

- $h / 6 y_{d}$. La constante de resorte $c_{a}=1 / \delta(\mathrm{kg} / \mathrm{cm})$ está definida por la fuerza $P$, en $\mathrm{kg}$, que produce la deformación lateral de $1 \mathrm{~cm}$. Conociéndose las dimensiones del perfil y la longitud libre de la viga (distancia entre los apoyos o entre los mamparos transversales) puede calcularse la constante de resorte considerando el marco abierto y aplicando, por ejemplo, el principio del trabajo virtual. Para aumentar la resistencia al pandeo de este cordón se colocan mamparos transversales o arriostramientos laterales cuya distancia $l_{1}$ se calcula con la fórmula indicada en la figura 49.

Comparando las cuatro posibilidades de secciones de vigas (fig. 50), a saber: los perfiles $[]],[, \Pi$, puede verse que los perfiles [ y $]$ tiene la ventaja de tener una sola alma, pero la desventaja de no poseer un eje de simetría vertical. En el perfil [ el centro de esfuerzos cortantes no coincide con el de gravedad; mientras que en el perfil $Z$ existe esta coincidencia, pero tiene sus ejes principales inclinados. Los perfiles ¿ $\mathrm{y} \leftrightharpoons$ tienen dos almas, aumentando así el peso de acero, pero doble simetría, lo que permite un mejor aprovechamiento del acero a la flexión. El perfil $\square$ posee además una gran seguridad frente al pandeo lateral.

\section{Las uniomes}

Componiendo un perfil I con dos perfiles [ (con las almas lado a lado) debe calcularse la fuerza $P$ transmitida por las uniones, ejecutándose éstas con soldadura por puntos, rema-
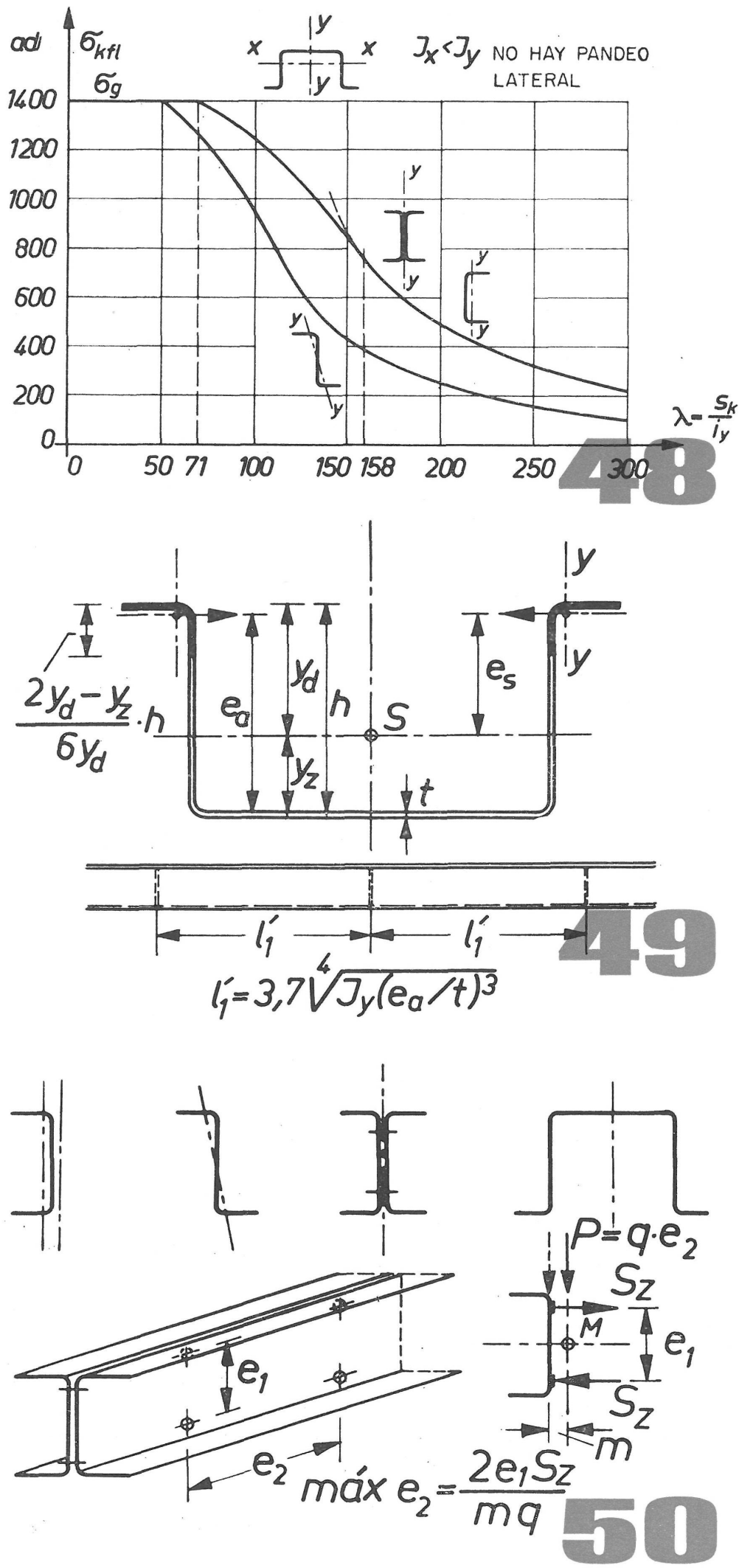
ches o tornillos. Evitando la torsión del perfil [ simple se tiene, con la fuerza admisible $S_{z}$ para cada una de las uniones, máx. $e_{2}=2 e_{1} S_{z} / m q$, siendo $q$ la carga distribuida uniformemente y $m$ la distancia del centro de esfuerzos cortantes al de gravedad (véase la figura 50 ). Teniendo cargas concentradas $P=P_{c}$, debe reducirse la distancia $e_{2}$ entre las uniones, en la zona correspondiente, a la tercera parte, para tener en cuenta la concentración local de las tensiones.

Para reforzar las vigas en las zonas de los momentos máximos se colocan platabandas, soldadas por puntos o por soldadura en ranura, en las alas del perfil formado en frío (fig. 51). Deben transmitirse a través de estas soldaduras los esfuerzos cortantes que actúan entre ala y platabanda. La distancia entre los puntos de soldadura se fija de tal manera que se evite el pandeo local de la platabanda. Siendo $\sigma$ la tensión normal en la platabanda, se tiene, para el acero de construcción St 37, la fórmula empírica:

$$
\text { máx.e }=[1.670 \times t / \sqrt{\sigma}] \mathrm{cm}<3 b,
$$

introduciéndose en esta fórmula $\sigma$, en $\mathrm{kg} / \mathrm{cm}^{2}$, y $t$, en $\mathrm{cm}$. El diagrama de la figura 51 da el esfuerzo cortante admisible, $T_{a d}$, en función del espesor de la platabanda. Ustedes pueden
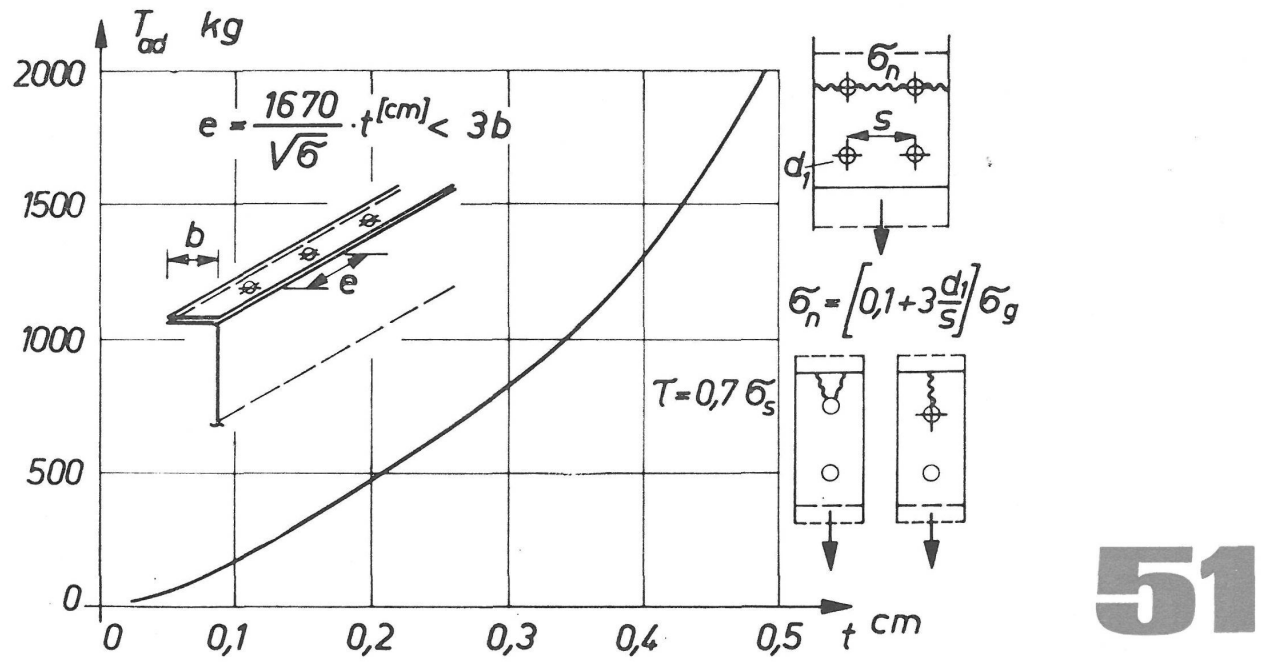

notar que $T_{a d}$ crece potencialmente con el espesor $t$ de la platabanda. Esto se debe al aumento de la distancia $e$ que aumenta también el peligro de pandeo local.

La distancia al borde del medio de unión (tornillos, remaches), se calcula teniendo en cuenta la resistencia al corte de la zona de borde (fig. 51). Existen dos posibilidades de fallo:

1) puede formarse una fisura en forma de cuña;

2) el perno puede cortar la chapa.

En el primer caso debe ser $e_{1} \leqq P / \sigma_{g} ; t_{\min } \geqq 1,5 d$, siendo $d$ el diámetro del perno; mientras que en el segundo caso la presión contra la pared del agujero no debe exceder de 4,8 $\sigma_{F} / 2,2$. Los ensayos han comprobado que la tensión neta admisible $\sigma_{n}$ depende de la distancia entre los agujeros (véase fig. 48), teniéndose con una distancia más pequeña una solicitación admisible $\sigma_{n}$ más grande. Esta solicitación se calcula con:

$$
\sigma_{n}=\left[0,1+3 d_{1} / S\right] \sigma_{g} .
$$


Se recomienda por esto colocar siempre los más tornillos o remaches posibles en una fila, perpendicularmente a la dirección de la fuerza.

\section{La wiga com allas anchas}

Consideremos, finalmente, la introducción de las tensiones en las alas anchas de vigas. Ya se sabe, desde hace mucho tiempo, que la distribución de las tensiones no es uniforme, a causa de la deformación tangencial de la rebanada de cabeza. E. Chwalla ha desarrollado fórmulas para calcular el «ancho eficaz» $b_{i}$, con el cual se obtiene la misma tensión máxima, suponiendo una distribución uniforme, que en la rebanada real. En la figura 52 está represen-

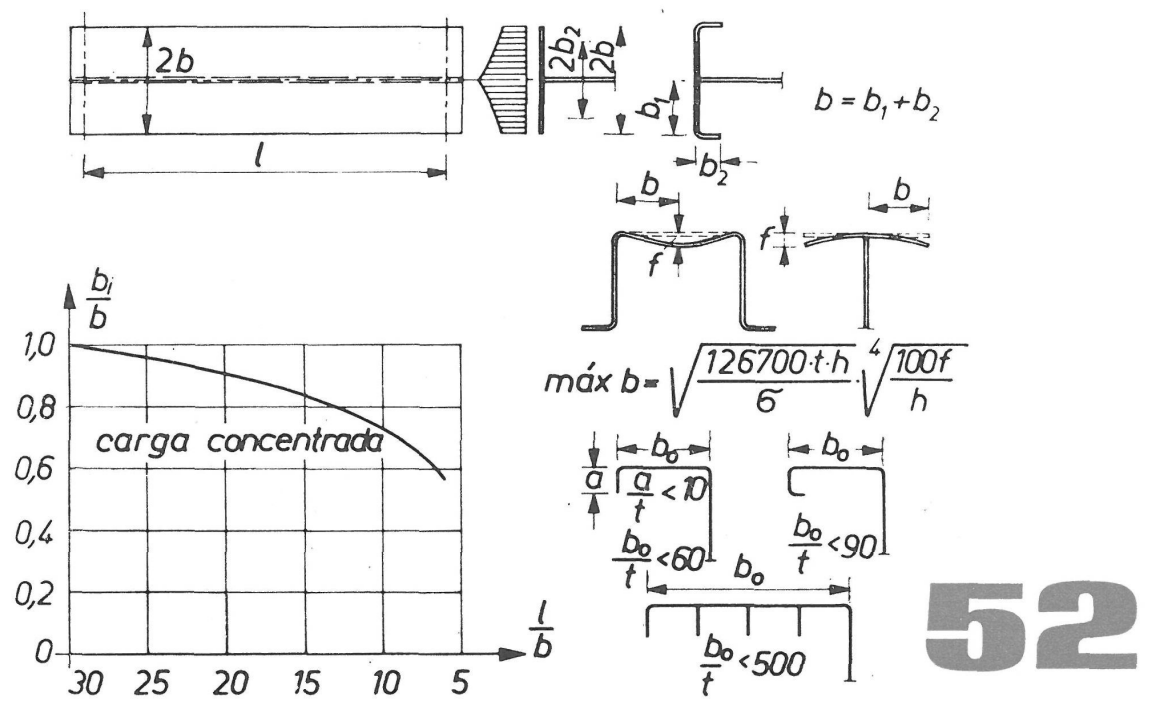

tado el diagrama $b_{i} / b$ en función de la relación $l / b$, siendo $b$ el ancho real del cordón y $l$ la luz de la viga. Se nota la reducción considerable de $b_{i} / b$, llegando a 0,5 , para $l / b=5$. Puede calcularse con el ancho total desde $l / b=30$.

El ancho real de la chapa de cabeza está limitado también por la deformación perpendicular al plano de la chapa, considerando el estado postcrítico (véase fig. 52). Admitiendo una cierta deformación, $f$, puede calcularse el ancho real máximo con la fórmula:

$$
\text { máx. } b=\sqrt{126.700 t \cdot h / \sigma} \times \sqrt[4]{100 f / h},
$$

siendo $h$ la altura del alma. Colocando labios en el borde libre de la chapa de cordón deben mantenerse las relaciones $b_{0} / t \leqq 60$ y $a / t \leqq 10$, aumentando el primer valor a 90 si se tiene un «labio plegado». Aunque se coloquen refuerzos intermedios, se recomienda no sobrepasar el valor $b / t=500$. Si se observan estas reglas, las deformaciones quedarán dentro de límites razonables. 